\title{
Damping rate and Lyapunov exponent of a Higgs field at high temperature
}

\author{
T.S. Biró ${ }^{1}$ and M.H. Thoma ${ }^{2}$ \\ ${ }^{1}$ MTA KFKI RMKI Theory Division, \\ H-1525 Budapest P.O.Box 49, Hungary \\ ${ }^{2}$ Institut für Theoretische Physik, Universität Giessen, \\ D-35392 Giessen, Germany
}

(June 30, 2021)

\begin{abstract}
The damping rate of a Higgs field at zero momentum is calculated using the Braaten-Pisarski method and compared to the Lyapunov exponent of the classical SU(2) Yang-Mills Higgs system.
\end{abstract}

PACS numbers: 11.15.Ha,12.90.+b

Typeset using REVTEX 


\section{INTRODUCTION}

Since the advent of gauge theories in the 1970-s there is a continous need for their application at high temperatures characteristic to high energy collisions of heavy nuclei or the early universe. Even asymptotically free theories, like QCD, prove to be far from trivial at high energy density or temperature.

Rather recently a promising method has been invented, the hard thermal loop (HTL) resummation [1], for treating field theories at high temperature consistently. This method is analytical, complementing extended numerical simulations on a space time lattice at finite temperature.

One of the most famous results of the HTL method, the gluon damping rate at zero momentum [2], was found to coincide with half of the Lyapunov exponent of a classical real-time lattice description of nonabelian gluon fields [3]. An attempt for understanding this at the first sight rather surprising agreement between two different quantities of high temperature gluon dynamics has been recently made by emphasizing that the main contribution to the gluon damping rate of order $g^{2} T$ stems from long wavelength gluons (i.e. from the infrared part of the gluon distribution) and hence can be a measure of the self-ergodizing speed of classically chaotic gluon fields [4].

In the present article we investigate whether a similar correspondence can be found in the coupled Yang-Mills Higgs-doublet system between the Higgs damping rate calculated in the HTL approximation and the Lyapunov exponent calculated on the lattice. If yes, it would let us surmise that something inherently classical is contained in the HTL method in the order of $g^{2} T$ and their results in principle could be simulated by classical dynamical calculations. If not, it would warn us that there is something uniquely particular for the pure gauge field systems. In fact chaos is opposite to damping, but because of time symmetry exponential growth and damping can have the same rate in given approximations. These are two facets of the same dynamics studied by using different boundary conditions in time.

Although the Yang-Mills-Higgs system has already been studied classically and found to 
be chaotic [3], for a throughout comparison with the new HTL results presented here we have to redo and extend that calculation, too.

\section{DAMPING RATES}

The aim of this section is the calculation of the Higgs and gauge boson damping rates at zero momentum and high temperature for comparing it with the Lyapunov exponent of the classical SU(2) Yang-Mills system coupled to a Higgs field [3]. We start from the Lagrangian

$$
\mathcal{L}=-\frac{1}{4} F_{\mu \nu}^{a} F_{a}^{\mu \nu}+\left(D_{\mu} \Phi\right)^{\dagger}\left(D^{\mu} \Phi\right)+\mu^{2} \Phi^{\dagger} \Phi-\lambda\left(\Phi^{\dagger} \Phi\right)^{2},
$$

where $\Phi$ is a charged Higgs doublet and $D_{\mu} \equiv \partial_{\mu}-g A_{a}^{\mu} T^{a}$ the covariant derivative containing the $\mathrm{SU}(2)$ generators $T^{a}$. At high temperatures above the phase transition $\Phi^{\dagger} \Phi \sim T^{2}$ and the quadratic term can be neglected besides the quartic term. Throughout this paper we consider $\mu=0$.

The damping rates follow from the imaginary part of the Higgs and gauge boson self energies, respectively. In order to obtain gauge invariant results, complete to leading order in the coupling constants, we adopt the Braaten-Pisarski method [1]. It is based on the distinction between hard momenta of the order of the temperature $T$ and soft ones of the order $g T$ or $\sqrt{\lambda} T$. The Braaten-Pisarski method amounts to the use of effective Green functions constructed by resumming the HTL diagrams. Calculating the gluon damping rate at zero momentum in this way solved the plasmon puzzle, a famous problem of finite temperature gauge theories [2].

The first step is to extract the HTL contributions to the effective Green functions, which arise from one-loop diagrams where the internal momenta are hard. Assuming all external momenta of the Green functions to be soft, the HTL corrections are of the same order in $g$ and $\lambda$ as the bare Green functions. In the case of a Yang-Mills field coupled to the Higgs field described by the Lagrangian (11) there are no HTL corrections to effective vertices involving

Higgs lines, as can be shown by power counting [1]. This observation holds also for scalar 
QED [5], which is apart from the gauge boson self coupling and $\mathrm{SU}(2)$ factors identical to (四), as well as for the Yukawa theory [6].

The HTL Higgs self energy, from which the effective Higgs propagator is constructed by resummation, follows from the diagrams of Fig.1, where the internal momenta of the polarization graph are hard. Adopting the usual Feynman rules [7], taking into account symmetry factors $1 / 2$ for the tadpole diagrams, we obtain the HTL Higgs self energy using the Matsubara formalism,

$$
\Xi_{\alpha \beta}=\delta_{\alpha \beta}\left(\frac{4}{3} \lambda+\frac{3}{16} g^{2}\right) T^{2}
$$

with the $\mathrm{SU}(2)$ indices $\alpha, \beta$ of the fundamental representation. As in the case of scalar QED [5] and the Yukawa theory [6] the HTL self energy is momentum independent and real.

The effective Higgs propagator is given by resumming the HTL self energy (2) in a Dyson-Schwinger equation leading to

$$
\Delta_{\alpha \beta}^{\star}=\frac{\delta_{\alpha \beta}}{K^{2}-m_{H}^{2}},
$$

where $K^{2}=k_{0}^{2}-k^{2}, k \equiv|\mathbf{k}|$ and $m_{H}^{2}=\left(4 \lambda / 3+3 g^{2} / 16\right) T^{2}$ is the square of the thermal Higgs mass.

The HTL gauge boson self energy is shown in Fig.2. The first three diagrams, containing the gauge boson self couplings and a ghost loop, give the well known results for the longitudinal and transverse parts of the self energies [8,9]

$$
\begin{aligned}
& \Pi_{L}^{a b}(K)=-3 \delta^{a b} m_{g}^{2}\left(1-\frac{k_{0}}{2 k} \ln \frac{k_{0}+k}{k_{0}-k}\right), \\
& \Pi_{T}^{a b}(K)=\frac{3}{2} \delta^{a b} m_{g}^{2} \frac{k_{0}^{2}}{k^{2}}\left[1-\left(1-\frac{k^{2}}{k_{0}^{2}}\right) \frac{k_{0}}{2 k} \ln \frac{k_{0}+k}{k_{0}-k}\right]
\end{aligned}
$$

with the thermal SU(2) gauge boson mass $m_{g}^{2}=2 g^{2} T^{2} / 9$ and the SU(2) indices $a, b$ of the adjoint representation. The remaining two diagrams involving a hard scalar loop show the same momentum and energy dependence as in (耳i) with a contribution to the thermal mass of $g^{2} T^{2} / 18$. In total the expression (四) is changed only by replacing $m_{g}^{2}$ by $m_{G}^{2}=5 g^{2} T^{2} / 18$.

The effective gauge boson propagator in Coulomb gauge is given by 


$$
\begin{aligned}
& D_{L}^{a b^{\star}}(K) \equiv D_{00}^{a b^{\star}}=\frac{\delta^{a b}}{k^{2}-\Pi_{L}(K)}, \\
& D_{T}^{a b^{\star}}(K) \equiv \frac{1}{2}\left(\delta_{i j}-\frac{k_{i} k_{j}}{k^{2}}\right) D_{i j}^{a b^{\star}}=\frac{\delta^{a b}}{K^{2}-\Pi_{T}(K)} .
\end{aligned}
$$

The Higgs damping rate at zero momentum is defined by

$$
\gamma_{H}(p=0)=-\frac{1}{4 m_{H}} \operatorname{Im} \Xi_{\alpha \alpha}^{\star}\left(\omega=m_{H}, p=0\right) .
$$

To leading order in the coupling constant $g$ the imaginary part of the Higgs self energy $\Xi^{\star}$ comes from the diagram in Fig.3. The damping mechanism can be read off from this diagram by cutting the internal lines [10]. The imaginary part of this diagram results from the discontinuous part of the effective gauge boson propagator (Landau damping) corresponding to the scattering of the soft Higgs off a thermal gauge boson or Higgs via the exchange of a soft gauge boson. (There is no pole-pole contribution due to kinematical reasons.) Owing to the absence of effective vertices and an imaginary part in the effective Higgs propagator there are no bremsstrahlung contributions, i.e. $2 \leftrightarrow 3$ processes, to the damping rate in leading order in contrast to the much more complicated damping rate of a soft gluon [2].

Since we consider the damping rate only at zero momentum, the transverse (magnetic) part of the gauge boson propagator does not contribute, leading to an infrared finite result due to Debye screening in the effective longitudinal (electric) propagator.

Using the Matsubara formalism we find

$$
\gamma_{H}(0)=\frac{3}{32 \pi} g^{2} T \int_{0}^{\infty} d k \frac{\left(\omega_{k}+m_{H}\right)^{3}}{\omega_{k}^{2}} \rho_{L}\left(\omega=\omega_{k}-m_{H}, k\right)
$$

where $\omega_{k}^{2}=k^{2}+m_{H}^{2}$ and

$$
\rho_{L}(\omega, k)=-\frac{1}{\pi} \operatorname{Im} D_{L}^{\star}(\omega, k)
$$

is the discontinuous part of the longitudinal gauge boson spectral function [11].

The remaining integral can be solved only numerically leading to Fig.4, where the Higgs damping rate $\gamma_{H} / g^{2} T$ is shown as a function of $\lambda / g^{2}$. The damping rate depends only weakly 
on the Higgs self interaction coupling $\lambda$, increasing from $0.018 g^{2} T$ at $\lambda=0$ to $0.029 g^{2} T$ if $\lambda$ tends to infinity.

The damping rate of the gauge boson at zero momentum is identical to the one calculated by Braaten and Pisarski [2]. There is no contribution from the polarization diagram by a Higgs pair due to kinematical reasons. Furthermore the final result is independent of the thermal gauge boson mass $m_{G}$. (Hence the gluon damping rate does not depend on the number of quark flavors in the quark-gluon plasma.) Therefore the gauge boson damping rate is given by the result in Ref. [2] with $N_{c}=2$, i.e.

$$
\gamma_{L, T}(0)=0.176 g^{2} T
$$

(The damping rates of a longitudinal and a transverse gauge boson are identical at zero momentum.) It should be noted that the gauge boson damping rate is about an order of magnitude larger than the one of a Higgs particle. This is caused partly by the fact that there is no bremsstrahlung contribution in the Higgs damping and partly by different $\mathrm{SU}(2)$ group factors.

Finally we would like to consider the damping rate of a hard Higgs particle with a momentum of the order of the temperature or larger. Although it is not related to the classical Lyapunov exponent, it might be of interest for cosmological problems, since it determines the behaviour of thermal and energetic Higgs particles, e.g. relaxation times and energy loss, in an electroweak plasma in or near equilibrium.

The hard damping rate follows from the same diagram (Fig.3) as the soft one. However, now it is sufficient to consider a bare Higgs propagator, i.e. setting $m_{H}=0$. Analogously to the case of a hard quark damping rate [12] we obtain an logarithmically infrared divergent result due to the absence of screening in the transverse part of the effective gauge boson propagator. Assuming an infrared cutoff of the order $g^{2} T$, as for example a magnetic screening mass [13] or by the Bloch-Nordsieck mechanism [14] we find to logarithmic accuracy

$$
\gamma_{H}(p \gtrsim T)=\frac{3 g^{2} T}{16 \pi} \ln \frac{1}{g}
$$




\section{LATTICE SIMULATION}

Now we turn to the computation of the Lyapunov exponent of the coupled Yang-Mills Higgs system starting from a classical real-time lattice description. The Hamiltonian of the continuum Higgs model,

$$
H=\frac{1}{2} E_{i}^{a} E_{i}^{a}+\frac{1}{4} F_{i j}^{a} F_{a}^{i j}+\dot{\Phi}^{\dagger} \dot{\Phi}+\left(D_{i} \Phi\right)^{\dagger}\left(D_{i} \Phi\right)-\mu^{2} \Phi^{\dagger} \Phi+\lambda\left(\Phi^{\dagger} \Phi\right)^{2},
$$

includes the complex Higgs doublet,

$$
\Phi=\frac{1}{\sqrt{2}}\left(\begin{array}{c}
-\Phi_{1}+i \Phi_{2} \\
\Phi_{3}+i \Phi_{0}
\end{array}\right)
$$

which can be represented by four real components also as a quaternion

$$
\Phi=\frac{1}{\sqrt{2}}\left(\Phi_{0}+i \tau^{c} \Phi^{c}\right)
$$

with $c=1,2,3$ and the $\tau$-s being the Pauli-matrices. These representations are related via the scalar product of $\mathrm{SU}(2)$ group elements $\langle A, B\rangle=\frac{1}{2} \operatorname{tr}\left(A B^{\dagger}\right)$

$$
\Phi^{\dagger} \Phi=\frac{1}{2}\left(\Phi_{0}^{2}+\Phi_{1}^{2}+\Phi_{2}^{2}+\Phi_{3}^{2}\right)=\frac{1}{2}\langle\Phi, \Phi\rangle .
$$

The Hamiltonian can be approximated on a spatial lattice with lattice spacing $a$ by using the variables

$$
\phi_{x}=\frac{a g}{2} \Phi(x)
$$

as quaternion variables on each lattice site $\left(x=0, \ldots, N^{3}-1\right)$ and

$$
U_{x, i}=e^{i \frac{a g}{2} \tau^{c} A_{i}^{c}(x)}
$$

as unit length quaternions $(\langle U, U\rangle=1)$ on each lattice link from $x$ to $x+i,(i=0,1,2)$. The canonically conjugate momenta of these lattice variables are

$$
P_{x, i}=\frac{4 a}{g^{2}} \dot{U}_{x, i} \quad \text { and } \quad \psi_{x}=\frac{4 a}{g^{2}} \dot{\phi}_{x}
$$


where the dot signals time derivation. The lattice Hamiltonian is 15

$$
\begin{aligned}
H= & \frac{g^{2}}{4 a}\left(\sum_{x} \frac{1}{2}\left\langle\psi_{x}, \psi_{x}\right\rangle+\sum_{x, i} \frac{1}{2}\left\langle P_{x, i}, P_{x, i}\right\rangle\right)+ \\
& \frac{4}{g^{2} a}\left(\sum_{x}\left(3-\frac{m_{f}}{2}\right)\left\langle\phi_{x}, \phi_{x}\right\rangle+\frac{g_{f}}{4}\left\langle\phi_{x}, \phi_{x}\right\rangle^{2}+\sum_{x, i} 1-\left\langle U_{x, i}, \frac{1}{4} V_{x, i}+\phi_{x} \phi_{x+i}^{\dagger}\right\rangle\right)
\end{aligned}
$$

Here $m_{f}=a^{2} \mu^{2}$ and $g_{f}=4 \lambda / g^{2}$ with $a$ being the lattice spacing. We used the following lattice approximation of the covariant derivative of the Higgs field

$$
a \cdot\left(D_{i} \phi_{x}\right)=U_{x, i} \phi_{x+i}-\phi_{x}
$$

and the identity

$$
\sum_{x, i}\left\langle U_{x, i} \phi_{x+i}, U_{x, i} \phi_{x+i}\right\rangle=\sum_{x, i}\left\langle\phi_{x}, \phi_{x}\right\rangle
$$

Now scaling the time to $t^{\prime}=t / a$ and the energy to $H^{\prime}=a g^{2} H / 4$ leads to

$$
\begin{aligned}
H^{\prime} & =\sum_{x} \frac{1}{2}\left\langle\psi_{x}, \psi_{x}\right\rangle+\left(3-\frac{m_{f}}{2}\right)\left\langle\phi_{x}, \phi_{x}\right\rangle+\frac{g_{f}}{4}\left\langle\phi_{x}, \phi_{x}\right\rangle^{2} \\
& +\sum_{x, i} \frac{1}{2}\left\langle P_{x, i}, P_{x, i}\right\rangle+1-\left\langle U_{x, i}, \frac{1}{4} V_{x, i}+\phi_{x} \phi_{x+i}^{\dagger}\right\rangle .
\end{aligned}
$$

Here and above the complement link variable, $V_{x, i}$, is constructed by adding triple products of group elements, $U$, on oriented links closing elementary plaquettes with the chosen link $(x, i)$ such that

$$
\sum_{x, i j} \frac{1}{2} \operatorname{tr} U_{x, i j}=\sum_{x, i j}\left\langle U_{x, i j}, 1\right\rangle=\frac{1}{4} \sum_{x, i}\left\langle U_{x, i}, V_{x, i}\right\rangle .
$$

Here $U_{x, i j}$, the plaquette variable is the product of the four link variables $U$ circumventing the plaquette with corner $x$ and lying in the $i j$ plane.

The scaled equations of motion are

$$
\begin{gathered}
\dot{U}_{x, i}=P_{x, i} \quad \dot{\phi}_{x}=\psi_{x} \\
\dot{P}_{x, i}=V_{x, i}+\phi_{x} \phi_{x+i}^{\dagger} \quad \dot{\psi}_{x}=W_{x}-F_{x}
\end{gathered}
$$


with

$$
W_{x}=\sum_{i} U_{x, i} \phi_{x+i}+U_{x-i, i}^{\dagger} \phi_{x-i}
$$

and

$$
F_{x}=\left(6-m_{f}+g_{f}\left\langle\phi_{x}, \phi_{x}\right\rangle\right) \phi_{x}
$$

A gauge transformation which leaves the lattice Hamiltonian (18) invariant and changes the lattice derivative (19) covariantly is given by

$$
\begin{aligned}
U_{x, i}^{\prime} & =g_{x}^{\dagger} U_{x, i} g_{x+i} \\
\phi_{x}^{\prime} & =g_{x}^{\dagger} \phi_{x}
\end{aligned}
$$

with $g_{x}$ being an arbitrary $\mathrm{SU}(2)$ group element. The static Noether charge corresponding to such time independent gauge transformations becomes

$$
\Gamma_{x}^{\prime}=\frac{g^{2}}{4} \Gamma_{x}=\sum_{i}\left(U_{x-i, i}^{\dagger} P_{x-i, i}-P_{x, i} U_{x, i}^{\dagger}\right)-\psi_{x} \phi_{x}^{\dagger}
$$

In order to relate the numerical simulation to physical parameters we consider the average energy density

$$
\bar{\varepsilon}=\frac{1}{a^{3} N^{3}} \frac{4}{a g^{2}} H^{\prime}=\frac{12}{a^{4} g^{2}}\left(\frac{1}{3 N^{3}} H^{\prime}\right)
$$

The expression in the last brackets is the scaled energy per link on an $N \times N \times N$ periodic lattice. Comparing it with the energy density of an ideal gas of SU(2) gluons and Higgs bosons at a fixed temperature $T$, i.e. with $\epsilon=\pi^{2} T^{4} / 3$ we arrive at

$$
\frac{1}{3 N^{3}} H^{\prime}=\frac{\pi^{3}}{9} \alpha_{w}(a T)^{4}
$$

When obtaining the equivalent temperature the Higgs fields are also considered, since - as the results of the numerical simulations - the energy is equally partitioned between electric magnetic and rest degrees of freedom in the final, chaotized state. $\alpha_{w}=g^{2} / 4 \pi$ is the weak fine structure constant which can be renormalized at high temperature perturbatively. Its 
known dependence on the physical temperature (via the perturbative beta function) sets the physical scale in the originally scaleless classical simulation.

Finally we note that in order to ensure the unitarity of the group elements $\langle U, U\rangle=1$ and its conservation $\langle U, P\rangle=0$ during the solution of the equations of motion Lagrange multipliers must be used. Including a small scaled timestep factor $d t / a=h$ into the conjugate momenta and its square into the respective forces we arrive at the following implicit recursion scheme, which conserves the static Noether charge 16]

$$
\begin{aligned}
& U^{\prime}=U+\left(P^{\prime}-\varepsilon U\right) \\
& P^{\prime}=P+\left(V-\mu U+\varepsilon P^{\prime}\right) \\
& \phi^{\prime}=\phi+\psi^{\prime} \\
& \psi^{\prime}=\psi+(W-F)
\end{aligned}
$$

with $\mu=\left\langle P^{\prime}, P^{\prime}\right\rangle$ and $\varepsilon=\left\langle U, P^{\prime}\right\rangle$.

\section{LYAPUNOV EXPONENTS}

We use the following gauge invariant definition of the distance between two different gluon and Higgs field configurations, respectively

$$
\begin{aligned}
& D\left[U, U^{*}\right]=\sum_{x, i}\left|\langle U, V\rangle-\left\langle U^{*}, V^{*}\right\rangle\right|, \\
& D\left[\Phi, \Phi^{*}\right]=\sum_{x, i}\left|\langle\Phi, \Phi\rangle-\left\langle\Phi^{*}, \Phi^{*}\right\rangle\right| .
\end{aligned}
$$

We sampled parallel runs on an $N=10$ cubic lattice of randomly initialized $U, \Phi$ configurations with unit length. In the dynamical simulation the length of the Higgs field could change freely according to the dynamics while the length of the gauge field remained equal to one. We repeated each calculation with slightly rotated initial configuration $U^{*}, \Phi^{*}$. The time evolution of these initially small distances has been observed over a time long enough for the saturation. (Distances in a compact space tend to saturate.) 
As can be seen in Fig.5 the configurations diverge exponentially in the $U$ as well as in the $\Phi$ configuration space. In the linear range of this evolution the leading Lyapunov exponent can be extracted simply

$$
h=\frac{d}{d t} \ln D(t) .
$$

Choosing maximally randomized purely magnetic gauge field configurations and similar Higgs field configurations initially we studied the chaotic behavior due to the extraction of leading Lyapunov exponents for different values of $g_{f}=4 \lambda / g^{2}$. As a result of this study the Higgs and gauge boson Lyapunov exponents are plotted in Fig.6. As expected, the Lyapunov exponent of the gauge boson does not depend on $\lambda$ and agrees with twice of the damping rate (9) within the numerical accuracy.

Surprisingly the Higgs Lyapunov Exponent shows a behavior qualitatively and quantitatively different from that of the damping rate obtained in the resummed one loop approximation (Fig.4). We remind, that this is contrary to the case of the pure gauge field system, where the Lyapunov exponent has been found to be twice of the gluon damping rate at zero momentum [4].

The result of the classical lattice dynamics can be understood easily: for very high values of $\lambda$ the $\Phi$-fields are constrained to keep a rather definite value of $\langle\Phi, \Phi\rangle$. The number of light degrees of freedom is reduced from four to three, so the Higgs sector becomes less chaotic. In the simulations presented here the gauge fields were initialized with random phases covering the whole group $\mathrm{SU}(2)$ uniformly in the magnetic sector, so these degrees of freedom could not take any energy from the Higgs fields over. The electric sector was able to receive energy also from the Higgs, but because of the final equipartition with the magnetic degrees of freedom the total energy converted into chaotic motion remained limited.

From the results of the numerical simulation presented here we must conclude that the Higgs damping rate, contrary to the gluon damping, is not closely related to the chaotic behavior of the classical fields. While the chaotic behavior of Higgs fields is rather set by the damping rate of gluons which couple to the Higgs covariantly and decreases somewhat at 
high $\lambda$ values, their resummed damping rate at high temperature increases with increasing Higgs-self coupling due to the thermal Higgs-mass which sets the scale in that calculation (可).

\section{ACKNOWLEDGMENTS}

M.H.T. thanks E. Braaten and A.K. Rebhan for helpful discussions. This work was supported by the Deutsche Forschungsgemeinschaft and the Hungarian Academy of Sciences (DFG-MTA 79/1995), by the Hungarian National Scientific Research Fund (OTKA T014213) and by BMBF and GSI. T.S.B. thanks for the fellowship of the University of Bergen which enabled him to visit the Physics Department there and to carry out a part of the numerical calculations. 


\section{REFERENCES}

[1] E. Braaten and R.D. Pisarski, Nucl. Phys. B337, 569 (1990).

[2] E. Braaten and R.D. Pisarski, Phys. Rev. D 42, 2156 (1990).

[3] T.S. Biró, C. Gong, B. Müller, and A. Trayanov, Int. Jour. Mod. Phys. C 5, 113 (1994).

[4] T.S.Biró, C.Gong, and B.Müller, Phys. Rev. D 52, 1260 (1995).

[5] U. Kraemmer, A.K. Rebhan, and H. Schulz, Ann. Phys. (N.Y.) 238, 286 (1995).

[6] M.H. Thoma, Z. Phys. C 66, 491 (1995).

[7] C. Itzykson and J.-B. Zuber, Quantum Field Theory (McGraw-Hill, New York, 1980).

[8] H.A. Weldon, Phys. Rev. D 26, 1394 (1982).

[9] V.V. Klimov, Zh. Eksp. Teor. Fiz. 82, 336 (1982) [Sov. Phys. JETP 55, 199 (1982)].

[10] H.A. Weldon, Phys. Rev. D 28, 2007 (1983).

[11] R.D. Pisarski, Physica A158, 146 (1989).

[12] M.H. Thoma and M. Gyulassy, Nucl. Phys. B351, 491 (1991).

[13] R.D. Pisarski, Phys. Rev. Lett. 63, 1129 (1989).

[14] J. Blaizot and E. Iancu, Saclay Report No. T95/146, 1995 (unpublished).

[15] T.S. Biró, S.G.Matinyan, and B. Müller, Chaos and Gauge Field Theory (World Scientific, Singapore, 1995).

[16] T.S. Biró, Int. J. Mod. Phys. C 6, 327 (1995). 


\section{FIGURES}

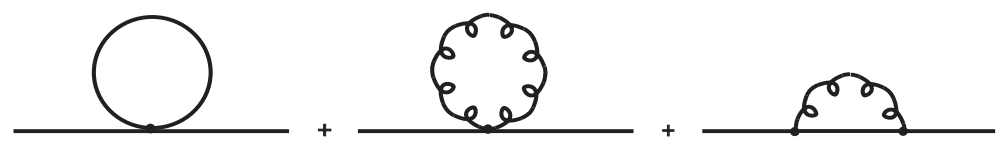

FIG. 1. HTL contributions to the Higgs self energy.
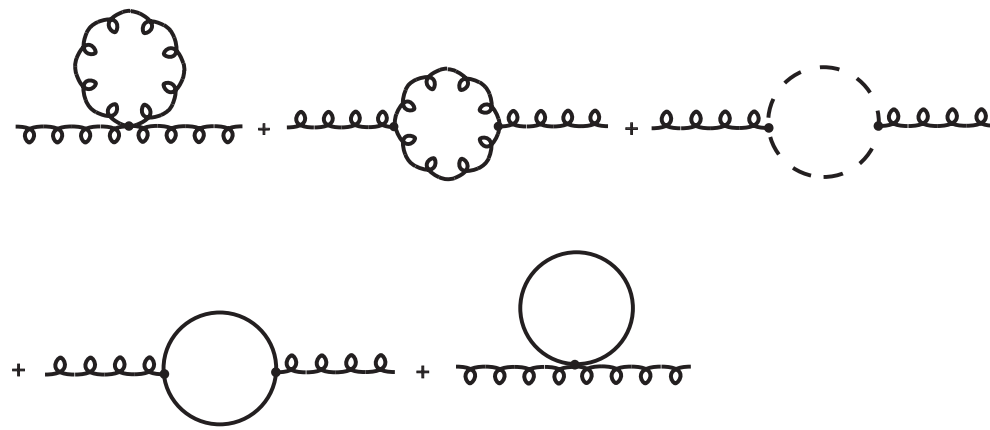

FIG. 2. HTL contributions to the gauge boson self energy.

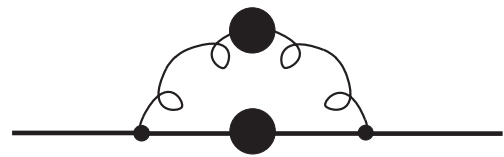

FIG. 3. Higgs self energy contribution to the damping rate.

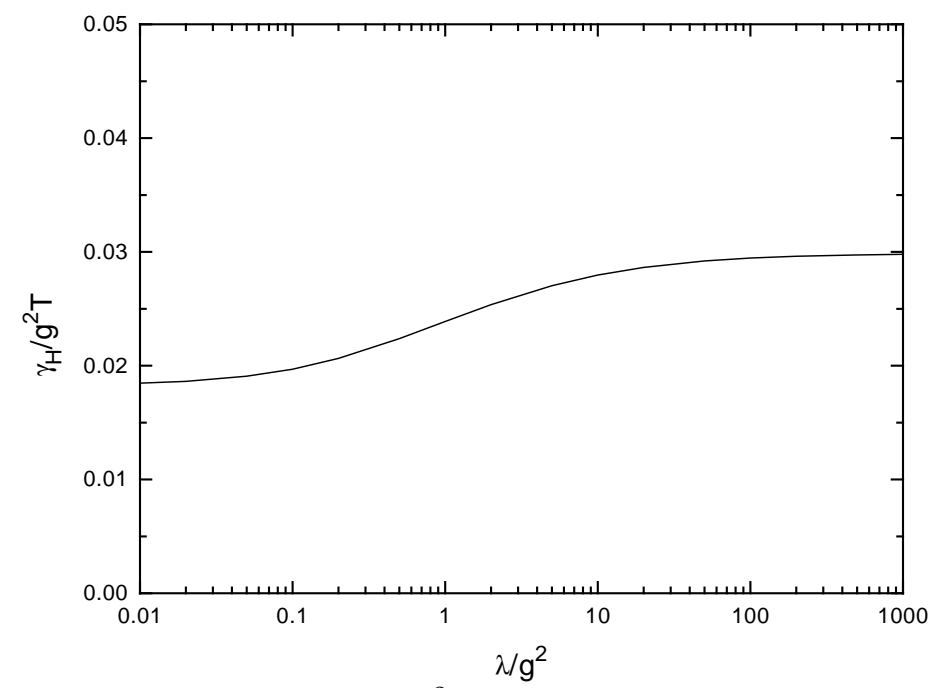

FIG. 4. Higgs damping rate $\gamma_{H} / g^{2} T$ at zero momentum as a function of $\lambda / g^{2}$. 


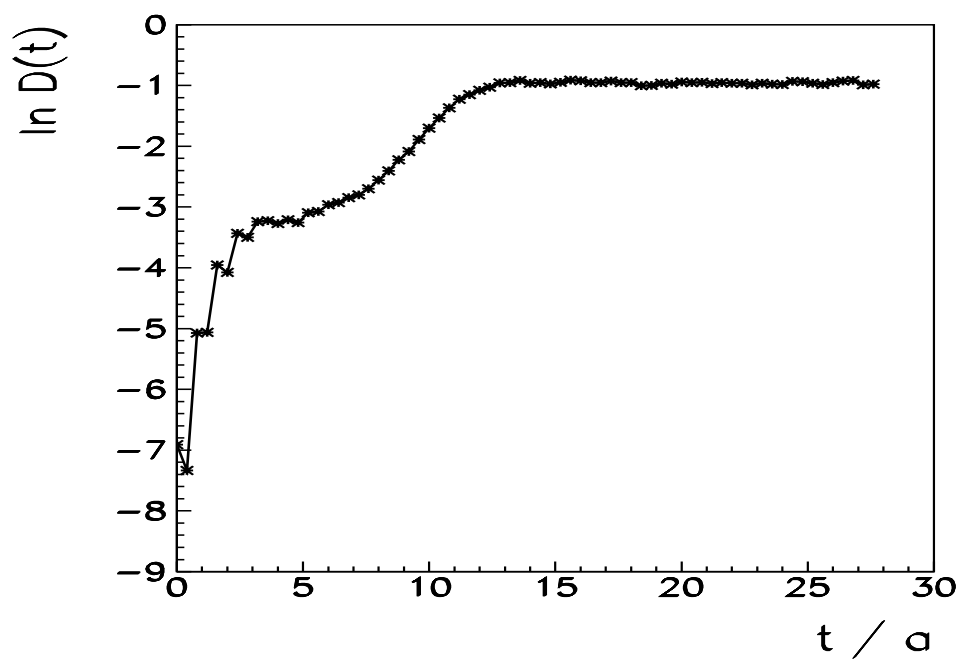

FIG. 5. Time evolution of the distance of two initially adjacent Higgs field configurations at $4 \lambda / g^{2}=1024$

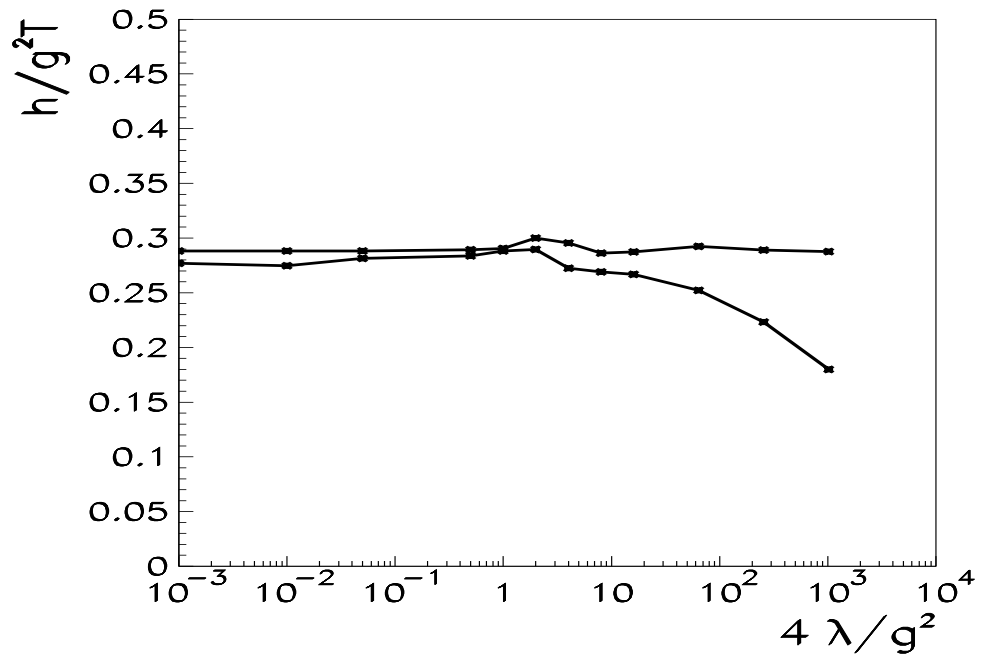

FIG. 6. The gauge field (upper curve) and Higgs field (lower curve) leading Lyapunov exponents as a function of $4 \lambda / g^{2}$ at high temperature. The numerical results have an error bar of about $20 \%$. 